\title{
Detección de fallas en máquinas rotatorias utilizando parámetros no lineales
}

\section{Fault detection in rotatory machinery using nonlinear parameters}

\author{
HUESCA-LAZCANO, Erick Eduardo $\dagger^{* *}$ FLORES-RAMIREZ, Oscar, ROMERO-RODRIGUEZ, \\ Gabriel y APAN-ARAUJO, Karla Cecilia
}

Universidad Politécnica de Amozoc, Ingeniería en Tecnologías de Manufactura

ID $1^{\text {er }}$ Autor: Erick Eduardo, Huesca-Lazcano / ORC ID: 0000-0002-0505-8442, Researcher ID Thomson: F-1162-2018, CVU CONACYT ID: 223342 ID $1^{\text {er }}$ Coautor: Oscar, Flores-Ramirez / ORC ID: 0000-0001-9884-9499, Researcher ID Thomson: E-8242-2018, CVU
CONACYT ID: 92914

ID $2^{\text {do }}$ Coautor: Gabriel, Romero-Rodriguez, / ORC ID: 0000-0002-5124-3016, Researcher ID Thomson: Y-2756-2018, CVU CONACYT ID: 299737

ID $3^{\text {er }}$ Coautor: Karla Cecilia, Apan-Araujo / ORC ID: 0000-0003-3373-226X, Researcher ID Thomson: E-8338-2018, CVU CONACYT ID: 896703

DOI: $10.35429 / J M E .2019 .12 .3 .25 .33$

Recibido 28 Agosto, 2019; Aceptado 30 Diciembre, 2019

\section{Resumen}

Con el desarrollo de la electrónica moderna y el incremento en el poder de procesamiento ahora es posible instalar muchos y diversos sensores en un solo tipo de maquinaria. Temperatura, vibración, presión, voltaje, etc. son variables que se monitorean comúnmente en maquinaria rotatoria. Estas variables en conjunto contienen toda la información relacionada con la condición de la máquina. En caso de una malfunción, esta, será reflejada en una o más de las variables monitoreadas. Estos cambios pueden ser tan sutiles que no pueden ser notados directamente en la serie temporal. Por lo tanto, es necesario transformar esta información en una nueva y más útil representación. En el presente trabajo se expone una metodología alternativa para el análisis y diagnóstico de malfunciones presentes en componentes de maquinaria rotatoria. Dicha metodología se basa en el procesamiento de las series temporales obtenidas de los sensores instalados en la maquinaria, sin considerar el modelo de esta. Se presenta como alternativa la extracción de parámetros no lineales entre los cuales destaca el máximo exponente de Lyapunov, como un indicador del estado de la máquina. En conjunto con parámetros tradicionales hace posible detectar fallas que son enmascaradas debido al comportamiento no lineal de los sistemas dinámicos.

Detección de fallas, Sistemas dinámicos, Exponente de Lyapunov

\begin{abstract}
With the development of modern electronics and the increase in processing power it is now possible to install many and diverse sensors in a single type of machinery. Temperature, vibration, pressure, voltage, etc. they are variables that are commonly monitored in rotating machinery. These variables together contain all the information related to the condition of the machine. In case of a malfunction, this will be reflected in one or more of the monitored variables. These changes can be so subtle that they can not be noticed directly in the time series. Therefore, it is necessary to transform this information into a new and more useful representation. In the present work an alternative methodology is exposed for the analysis and diagnosis of malfunctions present in components of rotating machinery. This methodology is based on the processing of time series obtained from the sensors installed in the machinery, without considering the model of this. The extraction of nonlinear parameters is presented as an alternative, among which the maximum exponent of Lyapunov stands out, as an indicator of the state of the machine. In conjunction with traditional parameters it makes it possible to detect faults masked due to non-linear behavior of dynamic systems.
\end{abstract}

Fault detection, dynamical systems, Lyapunov exponent

Citación: HUESCA-LAZCANO, Erick Eduardo, FLORES-RAMIREZ, Oscar, ROMERO-RODRIGUEZ, Gabriel y APANARAUJO, Karla Cecilia. Detección de fallas en máquinas rotatorias utilizando parámetros no lineales. Revista de Ingeniería Mecánica. 2019. 3-12: 25-33

\footnotetext{
* Correspondencia del Autor (erick.huesca@upamozoc.edu.mx)

$\dagger$ Investigador contribuyendo como primer Autor
} 


\section{Introducción}

La maquinaria rotatoria es de los equipos más utilizados en la industria. Actualmente el paradigma de mantenimiento es el que está basado en la condición, esto permite incrementar la disponibilidad del equipo, su seguridad, su eficiencia energética y su operación a bajo costo.

Una metodología más eficiente en el mantenimiento de maquinaria es el mantenimiento basado en el monitoreo de condición, en este se determina la condición actual de la maquinaria basado en la información proporcionada por distintos sensores los cuales puedes ser de temperatura, sonido, vibración, voltaje, corriente, etc

Los métodos actuales están basados en el análisis de las señales de vibración. Las señales de vibración dependen de varios factores como la carga de la maquinar, la velocidad de operación, la cimentación, la rigidez, etc. Al utilizar técnicas de procesamiento de señales se puede obtener información importante de la condición de la maquinaria, actualmente los métodos tradicionales se pueden dividir en métodos de análisis en el dominio del tiempo, análisis en el dominio de la frecuencia y análisis en dominio tiempo-frecuencia. Los métodos de análisis en el dominio del tiempo y frecuencia han resultado ser muy útiles para sistemas lineales los cuales proveen señales periódicas y estacionarias, pero para sistemas aún más complejos estos métodos carecen de buenos resultados. En estos últimos los métodos de análisis en el dominio del tiempo-frecuencia han resultado ser mas

Las señales de diferentes transductores proveen una serie temporal que contiene, en el caso de una malfunción, todos los síntomas relacionados a esta. En muchos casos no es fácil o incluso puede ser imposible distinguir la malfunción directamente en la serie temporal, debido a que la serie puede estar contaminada con ruido. Aun cuando existe la posibilidad de detectar la malfunción directamente en la serie temporal, si este fuera el caso, se necesitaría de personal técnico altamente calificado para analizar la información y poder diagnosticar la malfunción.
Para superar lo anterior, es posible manejar estos datos en un segundo paso conocido como extracción de parámetros característicos, en este paso es posible utilizar varias transformaciones de la entrada para producir nuevas salidas conocidas como parámetros característicos. Un parámetro característico es cualquier propiedad peculiar o cualidad, y podría ser simbólica o numérica.

\section{Marco Teórico}

\section{Desbalance}

El desbalance en la maquinaria rotatoria es una de las principales causas de vibración. La presencia de una excentricidad o una masa de desbalance en un disco rotatorio causa vibración, la cual puede ser aceptable hasta cierto nivel, la vibración causado por un desbalance que no es aceptable pude ser eliminando la masa excéntrica agregando una masa igual en una posición tal que cancele el efecto del desbalance. El desbalance en la maquinaria se atribuye a irregularidades tales como errores en el maquinado y variaciones en el tamaño de barrenos, tuercas, remaches, etc.

\section{Fisura}

Cuando se genera una fisura en la estructura del eje de una máquina rotatoria, la forma de esta es impredecible. Sin embargo, la existencia de una fisura en un eje rotatorio necesitar ser modelada para poder estudiar su evolución $\mathrm{y}$ comportamiento. Fundamentalmente se trabaja con dos tipos de fisuras, las de frente plano, o recto y las de frente elíptico. En la figura se pueden ver ambos tipos.

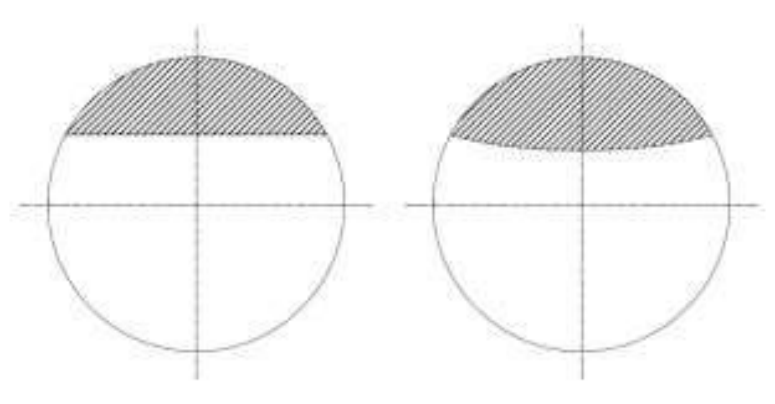

Figura 1 Tipos de frente de fisura

Fuente:

http://revistasomim.net/congreso2017/articulos/A1_87.p $d f$ 
Los sistemas rotatorios en estudio se consideran dominados por el peso, es decir, es la gravedad la que provoca que la fisura en el rotor se abrir y cerrar su posición angular cambie. Se sabe que la orientación de la fisura depende del tiempo, y cambia de forma periódica durante cada ciclo de rotación del eje, y es por eso que la fisura se abre y se cierra continuamente durante el giro del motor, a este fenómeno de apertura y cierre de la fisura se le conoce como respiro de la fisura.

\section{Reconstrucción del espacio de estados}

En el análisis de los sistemas dinámicos se utiliza frecuentemente el espacio de fases o espacio de estados para representar el comportamiento del sistema, consiste en la construcción de un espacio que tiene tantas dimensiones como el número de variables necesarias para especificar el estado del sistema original. Cada eje coordenado de este espacio representa una de las variables que componen el sistema.

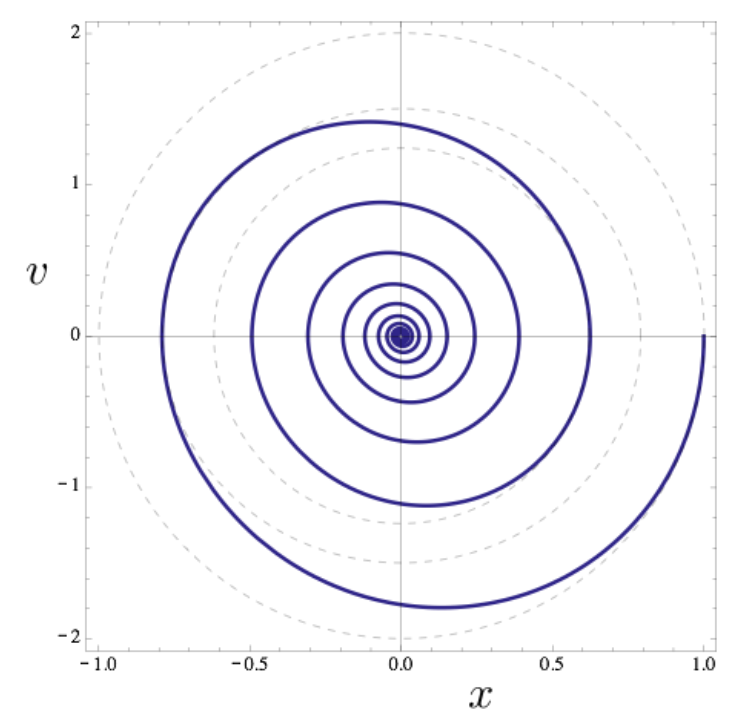

Figura 2 Espacio de estados

Fuente:

http://www.entropy.energy/scholar/node/dynamicalsystems-maps

En la figura 1 se muestra el espacio fase para el sistema dinámico péndulo simple amortiguado, en este caso $x_{1}$ representa la posición del péndulo y $x_{2}$ la velocidad. En esencia el espacio de estados representa el conjunto de posibles estados en los que puede estar el sistema modelado. El espacio de estados esta descrito por un campo vectorial que rige el recorrido de las variables del sistema en el tiempo, a este recorrido se le conoce como trayectoria.
Las trayectorias del sistema permiten realizar una descripción cualitativa de la evolución temporal del sistema que se está modelando. El acceso a todas las variables que gobiernan la dinámica de un sistema bajo investigación es poco probable en el mundo real. Sin embargo, tan solo una de serie temporal de los datos medidos de un sistema es afectada por todas las demás variables dinámicas relevantes y por lo tanto esta debe contener información histórica de la dinámica. Takens demostró que es posible reconstruir de manera cualitativa la dinámica del sistema en un espacio de estados ddimensional basado únicamente en la observación de una serie temporal escalar:

$x(t)=\left[x_{1}, x_{2}, \ldots x_{N}\right]^{T}$

usando el teorema de reconstrucción. Por lo tanto, el teorema de reconstrucción supone que tan solo un estado del sistema tiene la suficiente información acerca de los demás estados, y este espacio reconstruido preserva las propiedades topológicas del espacio de estados original, es decir, la dimensión fractal, el exponente de Lyapunov, etc.

La reconstrucción consiste en utilizar el método de retardos, en donde construimos una matriz con la siguiente estructura:

$X=\left[x\left(t_{i}\right), x\left(t_{i}+\tau\right), \ldots, x\left(t_{i}+(m-1) \tau\right)\right]$

Ahora i toma valores desde 1 hasta $N-$ $(m-1) \tau, \tau$ es un parámetro que se tiene que seleccionar para la reconstrucción al igual que $m$, que se conoce como la dimensión del espacio reconstruido.

De acuerdo con el teorema de Takens, cuando este procedimiento se hace de la manera correcta, la dinámica reconstruida usando esta fórmula es equivalente a la dinámica del sistema original, en el sentido de que las características invariantes son preservadas.

\section{Selección apropiada del retardo}

Un retardo adecuado debe cumplir dos criterios: en primer lugar, debe ser lo suficientemente grande de tal manera que la información medida de la variable $x$ en el tiempo $t+\tau$ sea relevante y significativamente diferente de la información que ya se conoce en el tiempo $t$. 
Solo así será posible obtener la información acerca de las otras variables que influencias a la variable medida para poder reconstruir de manera satisfactoria el espacio de estados con una elección razonable de $m$. En general un retardo pequeño puede ser compensado con una dimensión mayor de reconstrucción. Por esta razón el teorema original de reconstrucción está formulado con respecto a $m$ y no dice nada acerca de $\tau$. En segundo lugar, $\tau$ no deber ser tan grande ya que la variable observada puede perder "memoria" de su estado inicial, si este es el caso, el espacio de estados reconstruido se observará aleatorio dado que consistirá en puntos no correlacionados.

Para seleccionar el retardo se ocupa la información mutua, esta es una medida no lineal usada para cuantificar las correlaciones lineales y no-lineales, la información mutua entre $x_{t} \mathrm{y}$ $x_{t+\tau}$ cuantifica la cantidad de información que tenemos acercado del estado $x_{t+\tau}$ asumiendo que conocemos el estado $x_{t}$

$$
\begin{aligned}
& I\left(x_{t}, x_{t+\tau}\right)= \\
& -\sum_{h=1}^{j} \sum_{k=1}^{j} P_{h, k}\left(x_{t}, x_{t+\tau}\right) \ln \left[\frac{P_{h, k}\left(x_{t}, x_{t+\tau}\right)}{P_{h}\left(x_{t}\right) P_{k}\left(x_{t+\tau}\right)}\right]
\end{aligned}
$$

Donde $\quad P_{h} \quad$ y $\quad P_{k} \quad$ denotan las probabilidades de que las variables $x_{t}$ y $x_{t+\tau}$ tomen un valor dentro del h-ésimo y k-ésimo intervalo respectivamente. $P_{h, k}$ es la probabilidad conjunta. Si las variables $x_{t}$ y $x_{t+\tau}$ son completamente independientes, esto significa que estas variables no están correlacionadas y por lo tanto $I\left(x_{t}, x_{t+\tau}\right)$ es cero.

\section{Selección de la dimensión}

El método para seleccionar la dimensión de reconstrucción se conoce como el método de los falsos vecinos cercanos, el objetivo de este método es encontrar una dimensión de reconstrucción $m$ en la cual todos los puntos que son falsos vecinos son eliminados. Los falsos vecinos son puntos que están cercanos debió al valor menor de $m$. Cuando $m$ es pequeño, puntos que están alejados en el espacio de estados original se acercan en el espacio de estados reconstruido por lo tanto estos puntos son los falsos vecinos. Al incrementar la dimensión $m$ y determinando los vecinos, se puede eliminar a los falsos vecinos.

\section{Exponente de Lyapunov}

El exponente de Lyapunov es un índice que permite medir las características dinámicas de un sistema no lineal, mide la convergencia o divergencia por medio de la razón exponencial de las órbitas adyacentes en el espacio fase del sistema.

De manera breve se describe como se calcula el máximo exponente de Lyapunov utilizando la reconstrucción del espacio de estados, en este espacio reconstruido se selecciona arbitrariamente algún estado $[x(t), x(t+\tau), \ldots, x(t+[m-1] \tau)], \quad$ a continuación se localiza el vecino más cercano, en el sentido euclidiano, al punto inicial $\left[x\left(t_{0}\right), x\left(t_{0}+\tau\right), \ldots, x\left(t_{0}+[m-1] \tau\right)\right], \quad \mathrm{y}$ denotamos la distancia entre estos dos puntos como $L\left(t_{0}\right)$ en un tiempo después $t_{1}$, la longitud inicial habrá evolucionado a la longitud $L^{\prime}\left(t_{1}\right)$. El elemento de longitud es propagado a través del atractor por un tiempo suficientemente corto de tal forma que solo la estructura a pequeña escala del atractor es examinada. Si el tiempo de evolución es demasiado largo podríamos ver que $L^{\prime}$ se contrae debido a que las dos trayectorias que la definen podrían pasar por una región de contracción del atractor. Por lo que podríamos obtener una estimación errónea de $\lambda$.

A continuación, buscamos un nuevo punto que satisfaga los siguientes dos criterios razonablemente bien: que su separación $L\left(t_{1}\right)$ desde la trayectoria de referencia sea pequeña y que la separación angular entre los elementos de longitud de evolución y de reemplazo sea pequeño. Este procedimiento es repetido hasta que la trayectoria de referencia ha atravesado el archivo de datos, en cuyo caso se estima

$\lambda=\frac{1}{t_{p}-t_{0}} \sum_{k=1}^{P} \ln \frac{L^{\prime}\left(t_{k}\right)}{L\left(t_{k-1}\right)}$

En donde $\mathrm{P}$ es el numero total de pasos de reemplazo. El tiempo de evolución $t_{k+1}-t_{k}$ se mantiene constante.

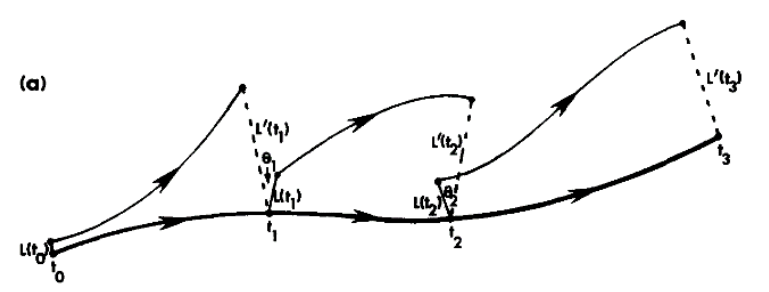

Figura 3 Cálculo de la distancia entre orbitas cercanas Fuente: https://hypertextbook.com/chaos/lyapunov-1/ 


\section{Plataforma experimental}

El rotor se encuentra montado sobre dos soportes rígidos, es decir sobre baleros de bolas. $\mathrm{La}$ energía de entrada la provee un motor controlado electrónicamente de la marca "Spectra Quest" que tiene un rango de operación hasta de 10000 RPM. Así mismo se utilizan sensores de la marca Bently Nevada de General Electric, mientras que para la adquisición de las señales se utiliza la plataforma CompactDAQ ${ }^{\circledR}$ de National Instruments. El rotor cuenta con un mecanismo que permite reproducir y controlar la apertura de la fisura y también permite modificar a voluntad el valor del ángulo entre el desbalance y el frente de la fisura.

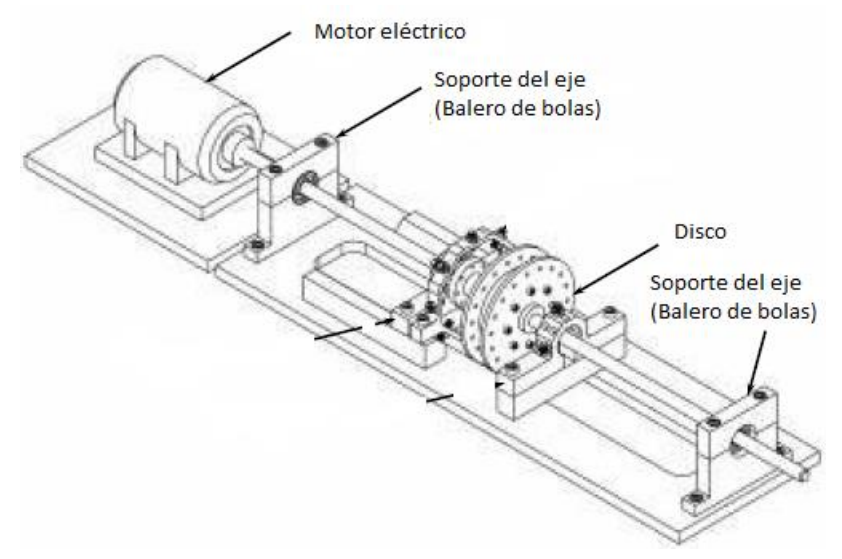

Figura 4 Plataforma experimental

Fuente: Laboratorio de vibraciones y rotodinámica ESIME-IPN

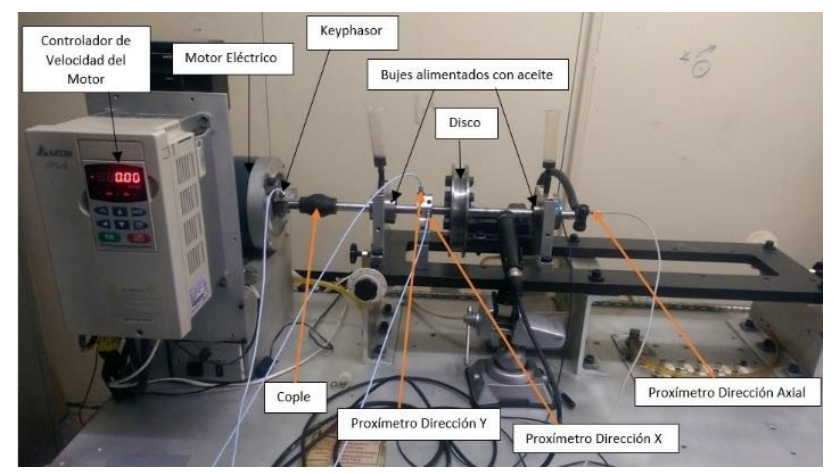

Figura 5 Plataforma experimental instrumentada

Fuente: Laboratorio de vibraciones y rotodinámica ESIME-IPN

En esta ocasión se utilizaron proxímetros como sensores de vibración, los proxímetros son de la marca Bently Nevada pertenecientes a la serie $3300 \mathrm{XL}$, entre sus principales características destaca su rango lineal de $1.5 \mathrm{~mm}$ a una distancia entre $0.25 \mathrm{~mm}$ y $1.75 \mathrm{~mm}$, su respuesta en frecuencia es de 0 a $10 \mathrm{kHz}$
Tomando como referencia el trabajo doctoral del Dr. Palacios, en el cual implementó un mecanismo que permite reproducir una fisura transversal sobre la superficie del rotor, el cual no solo permite controlar la profundidad de la fisura, sino que también permite modificar la posición angular entre el desbalance y el frente de la fisura.

A continuación, se presenta un par de fotografías con el detalle de este mecanismo, este mecanismo consta de un par de bridas unidas por doce tornillos cuidadosamente colocados en la periferia del disco.
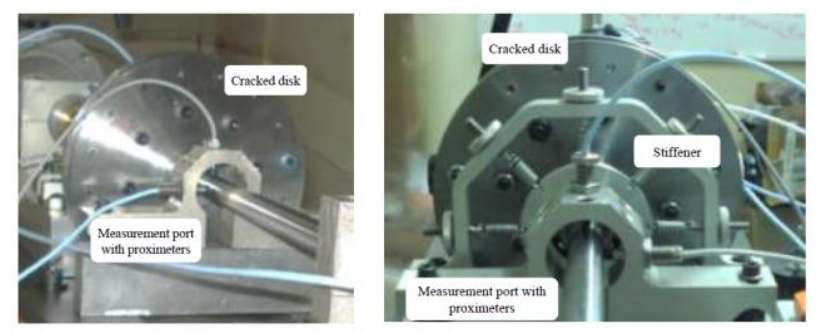

Figura 6 Detalles del disco central en el rotor que permite simular una fisura

Fuente: Laboratorio de vibraciones y rotodinámica ESIME-IPN

Se utilizaron doce tornillos en la brida que permite reproducir la grieta. El análisis de elemento finito realizado permite conocer la apertura de la fisura dependiendo del número y la disposición de los tornillos que era necesario aflojar para conseguir una apertura determinada.

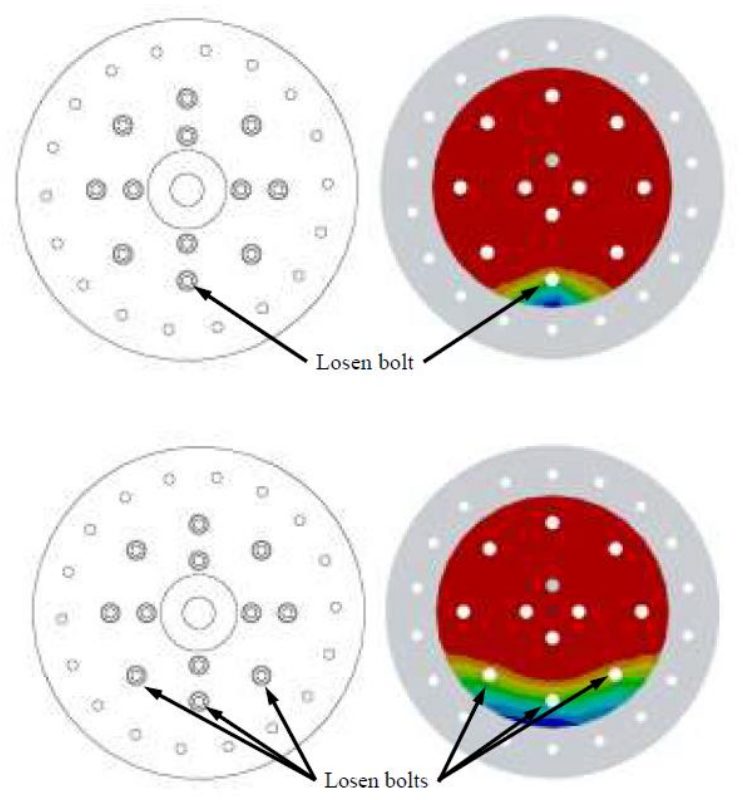

Figura 7 Influencia que tiene sobre la reproducción de la fisura la condición de apriete de algunos tornillos Fuente: http://revistasomim.net/congreso2017/articulos/A1_87.p $d f$

HUESCA-LAZCANO, Erick Eduardo, FLORES-RAMIREZ, Oscar, ROMERO-RODRIGUEZ, Gabriel y APAN-ARAUJO, Karla Cecilia. Detección de fallas en máquinas rotatorias utilizando parámetros no lineales. Revista de Ingeniería Mecánica. 2019. 


\section{Metodología propuesta}

La metodología propuesta se puede observar de forma general en el siguiente diagrama

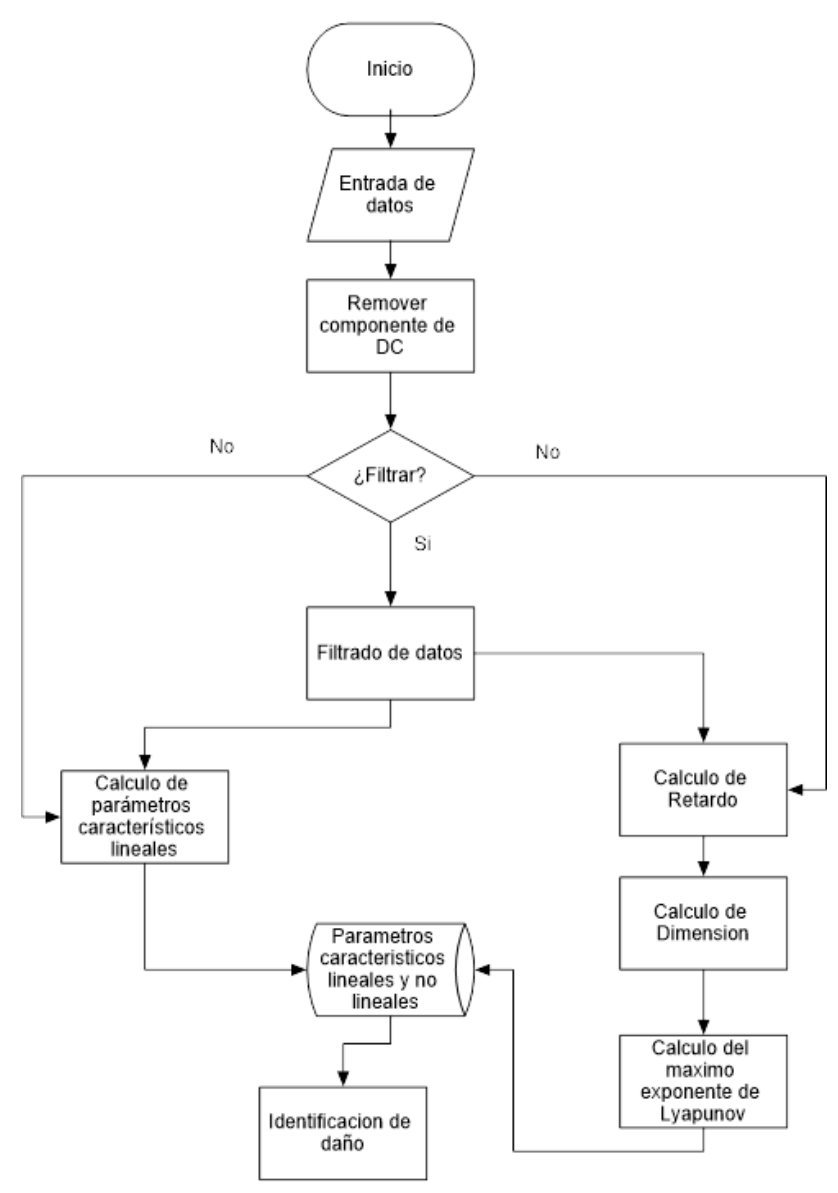

Figura 8 Diagrama de flujo de la metodología propuesta Fuente: Elaboración Propia

Como primer paso lo que se hace es remover la componente de DC, esto con el fin analizar solo el comportamiento vibratorio de la señal adquirida, es un paso muy simple que se resume en la siguiente expresión.

$\hat{x}=x-\bar{x}$

En donde $\bar{x}$ es el promedio de nuestra serie temporal. Después de remover la componente de DC, se propone el filtrado de los datos en caso de ser necesario, para los datos utilizados en este trabajo no se llevó a cabo este procedimiento. Para el calculo del retardo, aun cuando el método de la información mutua no dice nada acerca de como calcular el numero de intervalos para el calculo de las probabilidades es importante tener fundamentado la forma en que se van a calcular dichas probabilidades, en la literatura se proponen 3 reglas para conocer el numero de intervalos de nuestra señal, los cuales son:
- Regla de Sturge

$k=\left[\log _{2} N+1\right]$

- Regla de Scott

$k=\left[\frac{\max x-\min x}{3.5 s N^{-1 / 3}}\right]$

- Regla de Freedman-Diaconis

$k=\left[\frac{\max x-\min x}{2 Q N^{-1 / 3}}\right]$

La primera regla solo toma en consideración la longitud de la serie temporal y por lo tanto carece de la propiedad de adaptabilidad, por lo que esta regla se desecha. La regla de Scott toma como parámetro la desviación estándar, asumiendo que la distribución es gaussiana, mientras que la regla de Freedman-Diaconis no tiene ninguna suposición acerca de la distribución por lo que puede ser aplicada a cualquier tipo de distribución, por lo tanto, para el cálculo de probabilidades de la información mutua se toma esta regla.

Para el calculo de la dimensión se utiliza el ToolBox for Complex Systems y finalmente para el calculo del exponente de Lyapunov nos apoyamos del programa traducido a Matlab de Alan Wolf.

Se simularon 6 condiciones en la plataforma experimental, las cuales se resumen en la siguiente tabla:

\begin{tabular}{|l|l|}
\hline Condición & Nombre usado \\
\hline 1 tornillo flojo $\beta=0^{\circ}$ & Condición 1 \\
\hline 1 tornillo flojo $\beta=180^{\circ}$ & Condición 2 \\
\hline 1 tornillo flojo & Condición 3 \\
\hline 3 tornillo flojos $\beta=0^{\circ}$ & Condición 4 \\
\hline 3 tornillo flojos $\beta=180^{\circ}$ & Condición 5 \\
\hline 3 tornillos flojo & Condición 6 \\
\hline
\end{tabular}

Tabla 1 Condiciones experimentales Fuente: Elaboración Propia

La condición 3 y 6 contemplan solo la simulación de la falla de la fisura sin desbalance agregado, solo con el balance residual. La condición 1 y 2 simulan la combinación de las fallas fisura y desbalance la diferencia entre estas dos condiciones es la localización del desbalance con respecto a la fisura la cual se etiqueta con el ángulo $\beta$. 


\section{Resultados}

En primer lugar, se presenta la reconstrucción del espacio fase para las condiciones reproducidas en la plataforma experimental, es importante recalcar que las orbitas presentadas son construidas con una sola señal temporal adquirida de un proxímetro.

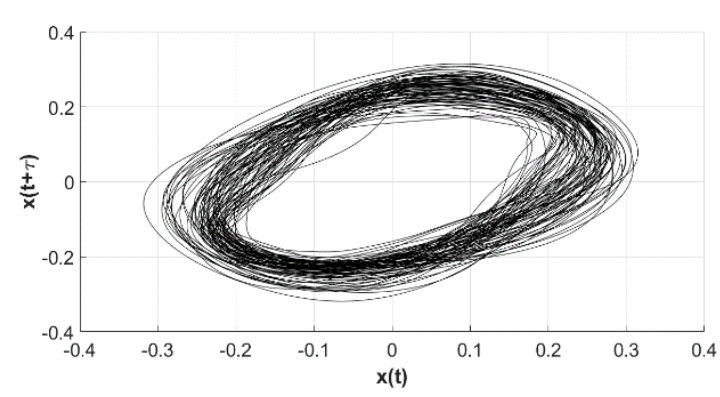

Gráfico 1 Espacio fase reconstruido para la condición 1 Fuente: Elaboración Propia

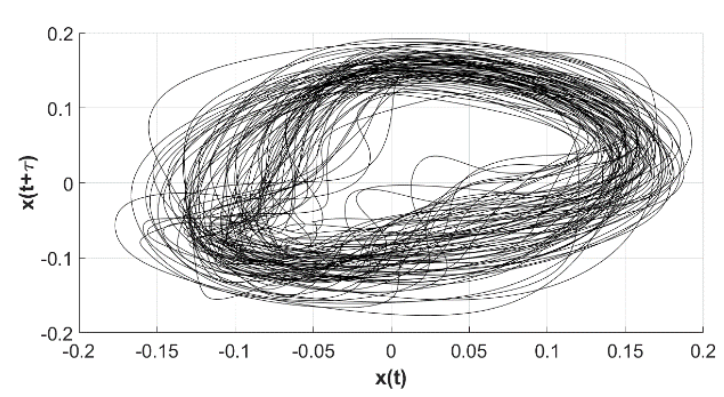

Gráfico 2 Espacio fase reconstruido para la condición 2 Fuente: Elaboración Propia

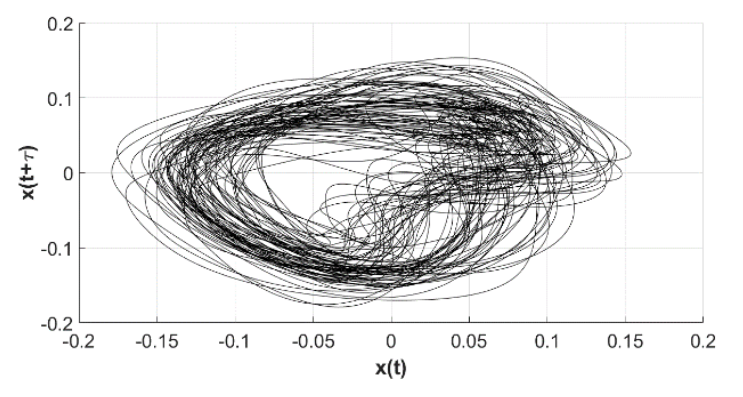

Gráfico 3 Espacio fase reconstruido para la condición 3 Fuente: Elaboración Propia

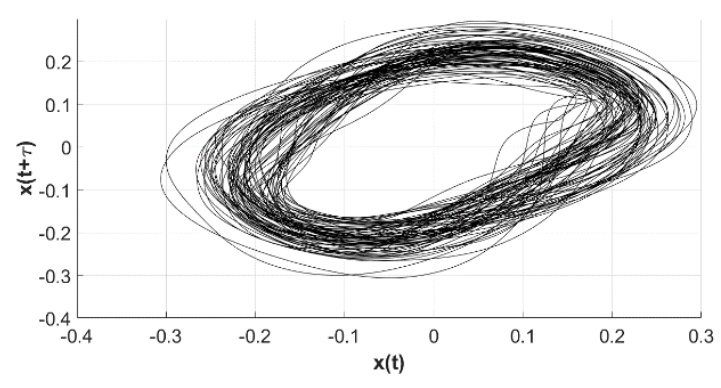

Gráfico 4 Espacio fase reconstruido para la condición 4 Fuente: Elaboración Propia

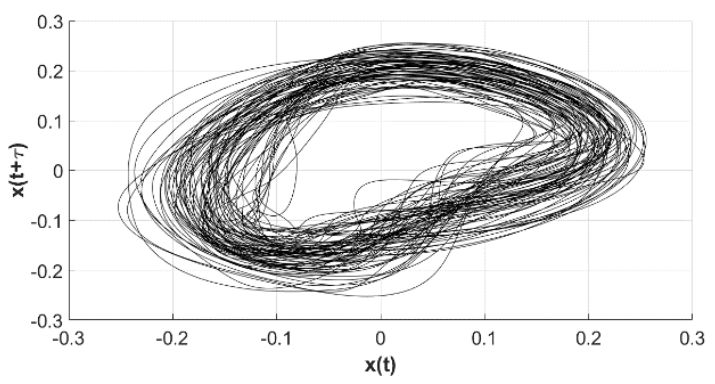

Gráfico 5 Espacio fase reconstruido para la condición 5 Fuente: Elaboración Propia

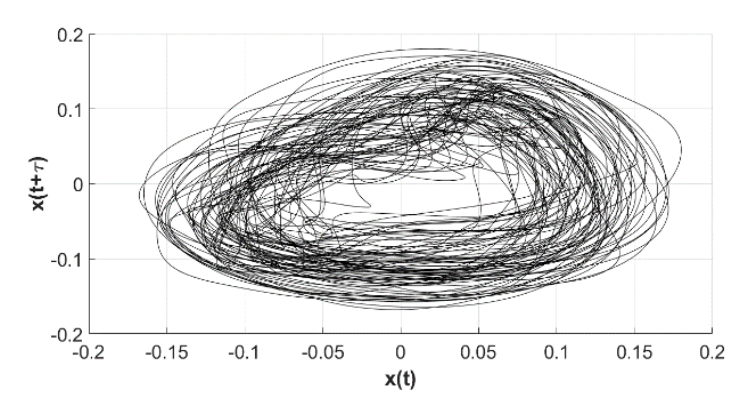

Gráfico 6 Espacio fase reconstruido para la condición 6 Fuente: Elaboración Propia

Como se puede observar en la reconstrucción del espacio de estados para la condición 1 y 4 se observan las órbitas con mayor amplitud, esto se explica fácilmente debido a que son las condiciones con el desbalance agregado, en contraste con la condición 2 y 5 que aun cuando también tiene desbalance se observa que su interacción con la fisura hace que la amplitud de las vibraciones no aumente demasiado. Por último, la condición 3 y 6 muestra el comportamiento del sistema solo con la falla fisura, nótese el comportamiento mas caótico de estas ultimas condiciones, prevaleciendo el comportamiento no lineal del sistema.

En el gráfico 7 se calcula el RMS de la señal de vibración, esta es una de las medidas más utilizadas en el monitoreo de maquinaria rotatoria, existe una clara disminución en los valores RMS de la vibración los cuales se logran al balancear el rotor, aun cuando se resuelve el problema del desbalance nuestra plataforma experimental sigue teniendo a la fisura presente, y el RMS es insensible a esta falla. 


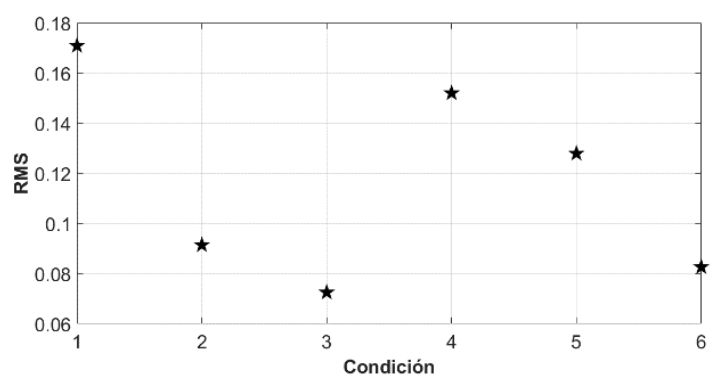

Gráfico 7 Valores RMS de las señales para las distintas condiciones

Fuente: Elaboración Propia

Si observamos el comportamiento del exponente de Lyapunov en el gráfico 8 , se observa que este cuantifica de mejor manera el comportamiento no lineal del sistema dinámico debido a la fisura, por lo tanto, en conjunto con el RMS podemos tener una mejor detección de fallas.

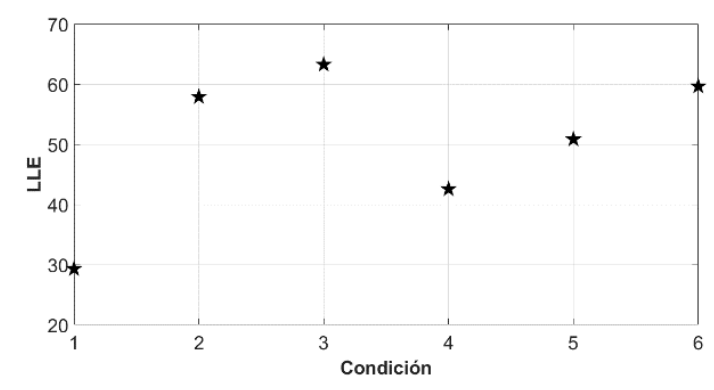

Gráfico 8 Máximo exponente de Lyapunov para las distintas condiciones

Fuente: Elaboración Propia

\section{Conclusiones}

Se ha realizado un análisis, sobre las fallas combinadas que pueden aparecer en maquinaria rotatoria. Este análisis considera como condiciones, la combinación de las fallas fisuradesbalance con distintas orientaciones. Se encontró que con parámetros tradicionales es posible que una falla de tan gran importancia como la fisura sea enmascarada debido al comportamiento no lineal del sistema dinámico, pero con el uso del máximo exponente de Lyapunov aún es posible detectarla.

La metodología propuesta tiene amplia aplicación y utilidad en el sector industrial y de servicios al poder evaluar, detectar daños y malfunciones incipientes de la operación de maquinaria rotatoria utilizando la extracción de parámetros no lineales

\section{Referencias}

Amirat, Y., Choqueuse, V., Benbouzid, M. E. H. (2010). Condition monitoring of wind turbines based on amplitude demodulation. Proceedings of the 2010 IEEE ECCE, 2417-2421.

Boudiaf, A., Moussaoui, A., Dahane, A., Atoui, I. (2016). A comparative study of various methods of bearing fault diagnosis using the Case Western Reserve University data. Journal of Failure Analysis and Prevention, 16(2), 271284.

Huamán, A., \& Antonio, A. (2019). Efecto de técnicas de mantenimiento predictivo en la detección temprana de fallas en los equipos rotativos de la empresa Ambev Peru sac.

Lu, B., Li, Y., Wu, X., Yang, Z. (2009). A review of recent advances in wind turbine condition monitoring and fault diagnosis. Proceedings of the 2009 IEEE PEMWA, 1-7.

Marwan, N., Thiel, M., Nowaczyk, N.R. (2002). Cross recurrence plot based synchronization of time series, Nonlin Proc. Geophys, 9, 325-331.

Palacios Pineda, Luis Manuel (2015). Diagnostico y prognosis en problemas dinámico/estructurales ocurridos en componentes de turbomaquinaria de alto desempeño.

Pater, T.H., Darpe, A.K. (2008). Influence of crack breathing model on nonlinear dynamics of a cracked rotor. Journal of sound and vibration. 311, 1953-1972.

Perc, M. (2006). Introducing nonlinear time series analisis in undergraduate courses. Fizika A. 15, 91-112.

Ronquillo, E., \& Douglas, C. (2019). Repotenciación del mantenimiento predictivo basado en el análisis de vibración enfocado a equipos rotatorios usados para el proceso de producción de una planta química ubicada en Guayaquil-Ecuador.

Santhana Raj, A., Murali, N. (2013). Early classification of bearing faults using morphological operators and fuzzy inference. IEEE Transactions on industrial electronics, $60(2), 567-574$. 
Sinou, J.J. (2009). A review of damage detection and health monitoring of mechanical systems from changes in the measurement of linear and non-linear vibrations. Mechanical vibrations: Measurement, Effects and Control, 643-702.

Tapia Montero, G. O. (2019). Diagnóstico de fallas en máquinas eléctricas de la planta BALMISA por análisis de Termografía.

Wolf, A., Swift, J.B., Swinney, H.L., Vastano, J. A. (1985). Determining Lyapunov exponents from a time series. Physica D, 16, 285. 